\title{
Space Launch System Mobile Launcher Modal Pretest Analysis
}

\author{
James C. Akers ${ }^{1}$ and Joel W. Sills Jr. ${ }^{2}$ \\ ${ }^{1}$ NASA Glenn Research Center \\ 21000 Brookpark Road \\ Cleveland, Ohio 44135 \\ ${ }^{2}$ NASA Johnson Space Center \\ 2101 NASA Parkway \\ Houston, TX 77058
}

\begin{abstract}
NASA is developing an expendable heavy lift launch vehicle capability, the Space Launch System, to support lunar and deep space exploration. To support this capability, an updated ground infrastructure is required including modifying an existing Mobile Launcher system. The Mobile Launcher is a very large heavy beam/truss steel structure designed to support the Space Launch System during its buildup and integration in the Vehicle Assembly Building, transportation from the Vehicle Assembly Building out to the launch pad, and provides the launch platform at the launch pad. The previous Saturn/Apollo and Space Shuttle programs had integrated vehicle ground vibration tests of their integrated launch vehicles performed with simulated free-free boundary conditions to experimentally anchor and validate structural and flight controls analysis models. For the Space Launch System program, the Mobile Launcher will be used as the modal test fixture for the ground vibration test of the first Space Launch System flight vehicle, Artemis 1, programmatically referred to as the integrated vehicle modal test. The integrated vehicle modal test of the Artemis 1 integrated launch vehicle will have its core and second stages unfueled while mounted to the Mobile Launcher while inside the Vehicle Assembly Building, which is currently scheduled for the summer of 2020. The Space Launch System program has implemented a building block approach for dynamic model validation. The modal test of the Mobile Launcher is an important part of this building block approach in supporting the integrated vehicle modal test since the Mobile Launcher will serve as a structurally dynamic test fixture whose modes will couple with the modes of the Artemis 1 integrated vehicle. The Mobile Launcher modal test will further support understanding the structural dynamics of the Mobile Launcher and Space Launch System during rollout to the launch pad, which will play a key role in better understanding and prediction of the rollout forces acting on the launch vehicle. The Mobile Launcher modal test is currently scheduled for the summer of 2019. Due to a very tight modal testing schedule, this independent Mobile Launcher modal pretest analysis has been performed to ensure there is a high likelihood of successfully completing the modal test (i.e. identify the primary target modes) using the planned instrumentation, shakers, and excitation types. This paper will discuss this Mobile Launcher modal pretest analysis for its three test configurations and the unique challenges faced due to the Mobile Launcher's size and weight, which are typically not faced when modal testing aerospace structures.
\end{abstract}

Keywords: Apollo, Artemis 1, Building Block Approach, Dynamic Test Fixture, Experimental Modal Analysis, Ground Vibration Test, Integrated vehicle Modal Test, Mobile Launcher, Modal Test, Rollout, Rollout Forces, Space Launch System, Space Shuttle, Vehicle Assembly Building

\section{BACKGROUND}

NASA is developing the expendable heavy lift launch vehicle, the Space Launch System (SLS), to support lunar and deep space exploration [1,2]. The Mobile Launcher (ML) is a very large and very heavy open beam/truss steel structure designed to support SLS during its buildup and integration in the Vehicle Assembly Building (VAB), transportation from the VAB out to the launch pad, and provides the launch platform at the launch pad. 
The Marshall Space Flight Center (MSFC) Test Stand (TS) 4550 was built in 1963, called the Advanced Saturn Dynamic Test Facility, and used for the Integrated Vehicle Ground Vibration Testing (IVGVT) for the Apollo Saturn V. Subsequently modified in 1975 for the GVT of the Space Shuttle and called the Space Shuttle Mated Ground Vibration Test Facility. Figure 1 shows the TS 4550 exterior and interior layout. Figure 2 shows the Saturn V Dynamic Test Vehicle, referred to as SA-500D, installed in Advanced Saturn Dynamic Test Facility (TS 4550). Figure 3 shows the installation of the Shuttle Orbiter Enterprise in the Space Shuttle Mated Ground Vibration Test Facility (TS 4550).
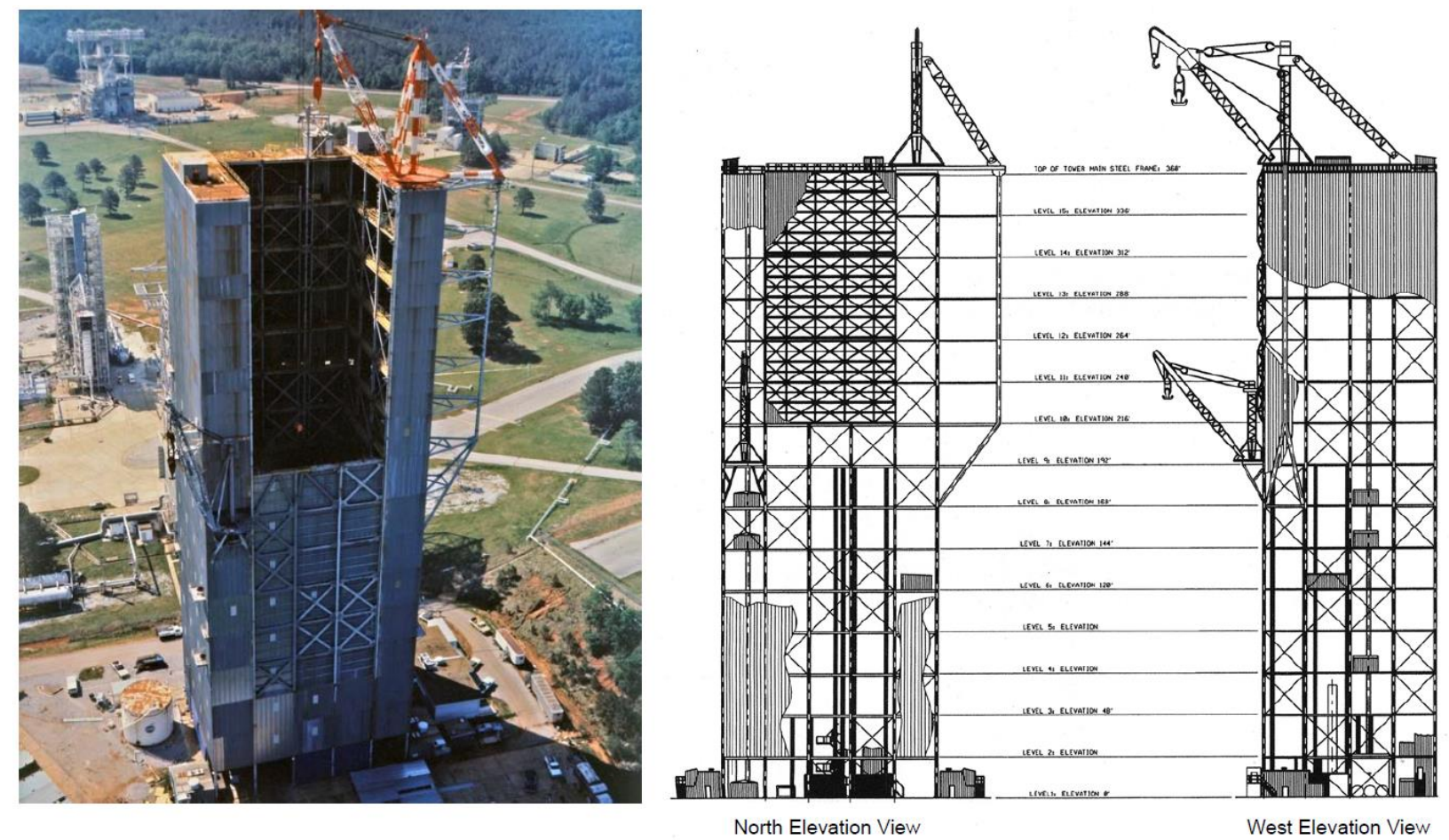

Figure 1. MSFC Test Stand 4550.

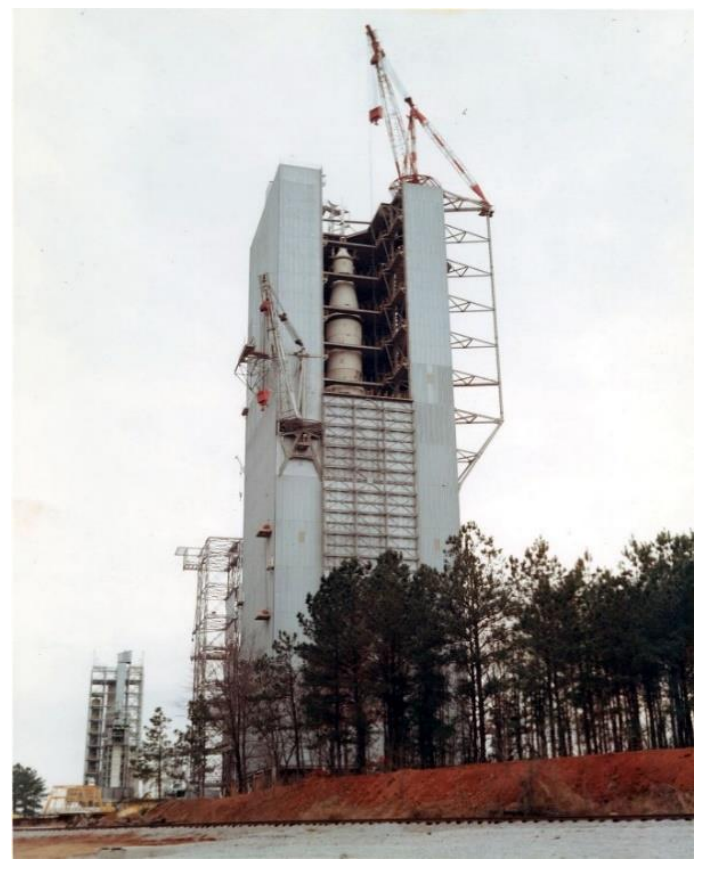

Figure 2. MSFC Advanced Saturn Dynamic Test Facility (Test Stand 4550) With Apollo SA-500D Installed. 


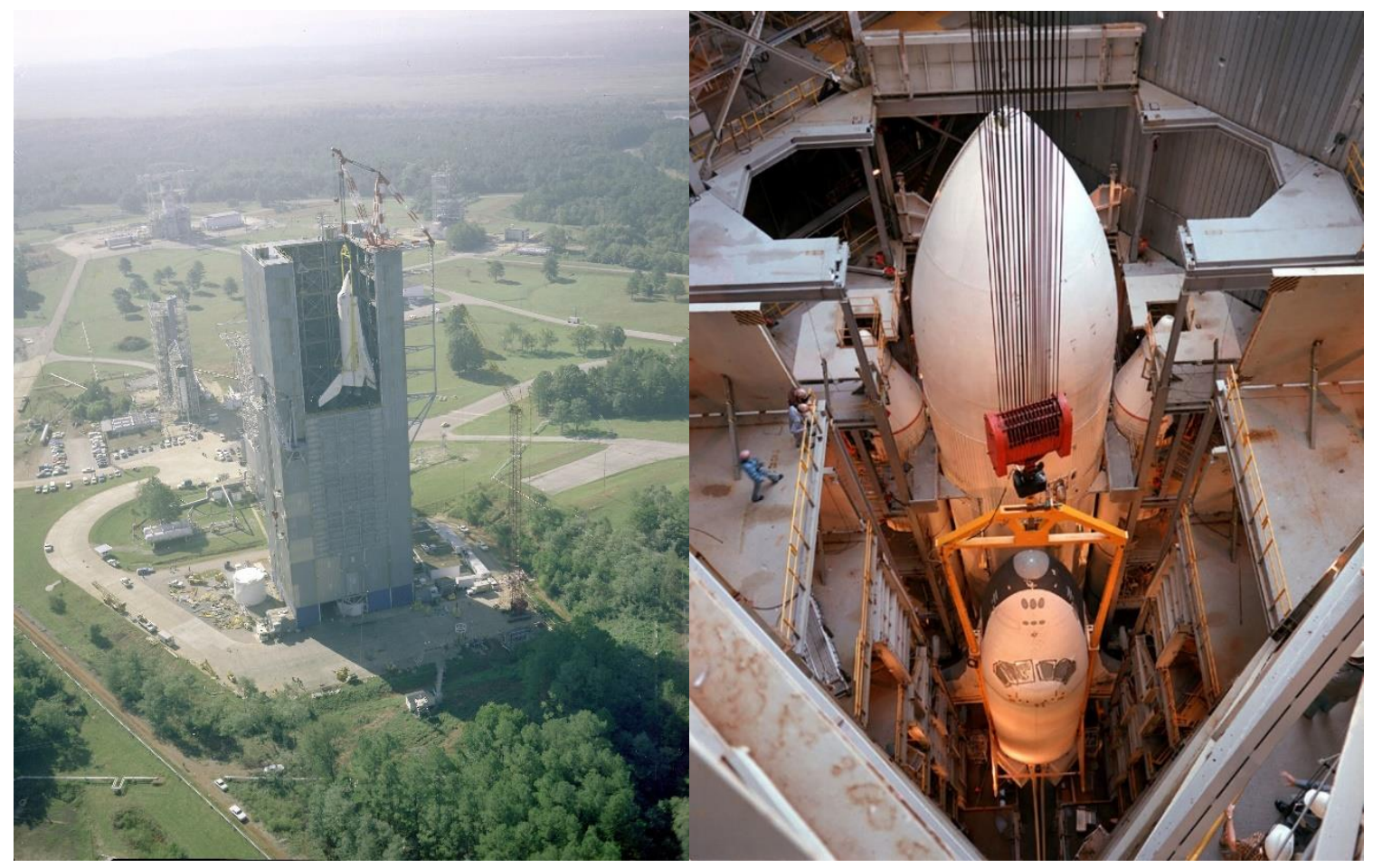

Figure 3. MSFC Space Shuttle Mated Ground Vibration Test Facility (Test Stand 4550) With Shuttle Orbiter Enterprise Being Lowered Into Place (1) And Being Integrated To The External Tank (r).

However, a programmatic decision was made to not refurbish TS 4550 and use it for the ground vibration test of the Artemis 1 integrated vehicle and referred to as the Integrated vehicle Modal Test (IMT). The Artemis 1 integrated vehicle consists of the SLS integrated with the Orion Multi-Purpose Crew Vehicle (MPCV) spacecraft and will have its core and its second stage, the Interim Cryogenic Propulsion Stage (iCPS), which is based upon the Delta IV Cryogenic Second Stage [7], unfueled.

The ML will serve as the IMT modal test fixture supporting the Artemis 1 integrated vehicle. The ML weighs over 10 million pounds and is over 360 feet tall $[8,9]$. The ML Deck supports the SLS at eight attachment points located at the bottom of its two boosters, which connect to the ML Vehicle Support Posts (VSP). The ML Tower provides lateral support to the integrated SLS launch vehicle via the Vehicle Stabilization System (VSS) and supports the fuel, power, and data umbilicals running to SLS and MPCV. The ML Tower also provides crew access to the MPCV Crew Module (CM). Figure 4 shows the Artemis 1 integrated vehicle on the ML. 

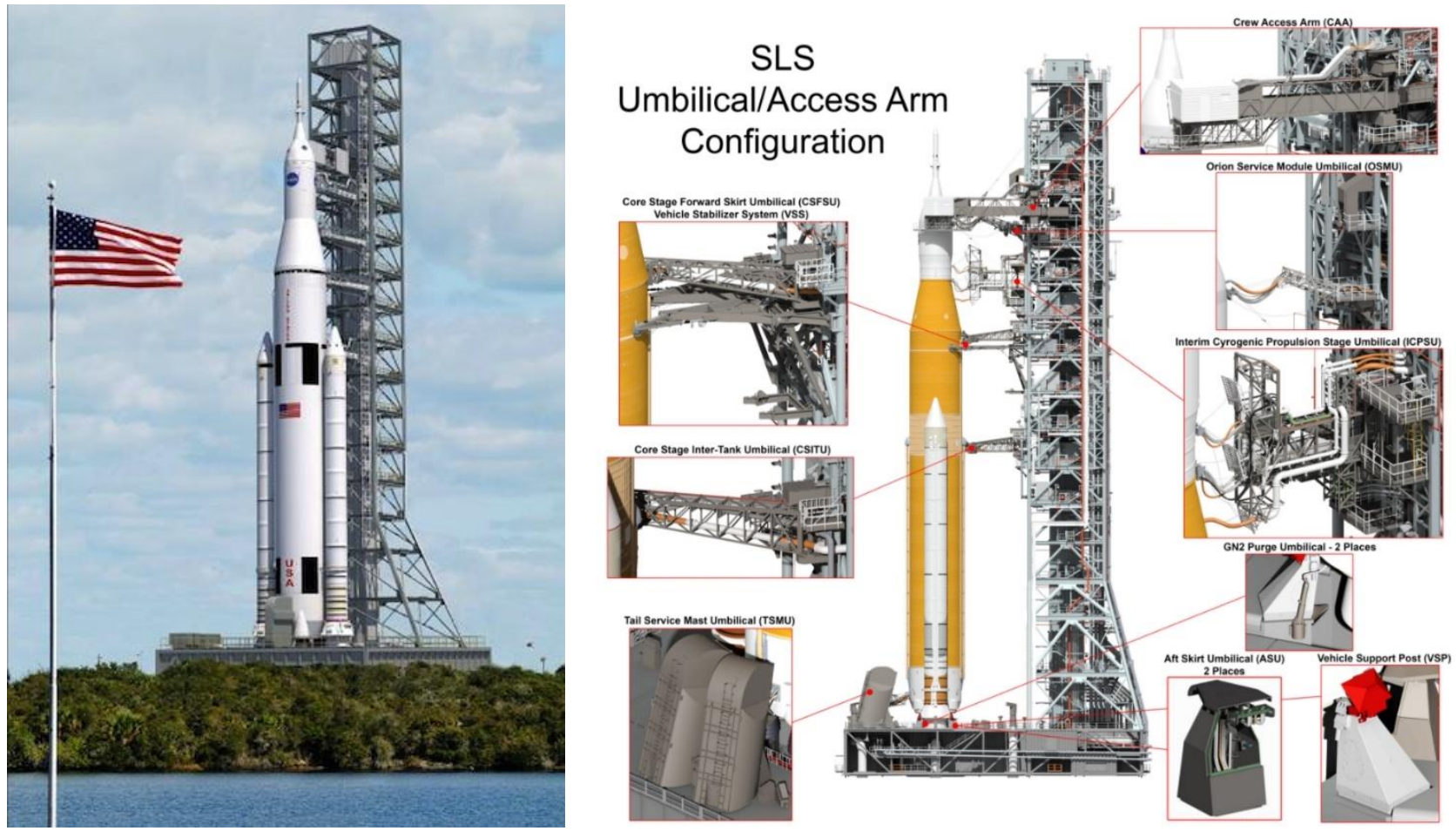

Figure 4. SLS On Mobile Launcher At Launch Pad (1) And Umbilicals (r), (from http://NASASpaceFlight.com).

The ML as the IMT modal test fixture presents unique technical challenges due to the ML providing a flexible boundary condition and its structural dynamics coupling with the Artemis 1 integrated vehicle. In addition, the ML is significantly heavier that the Artemis 1 integrated vehicle and therefore motion in the ML will end up "driving" responses in the Artemis 1 integrated vehicle. This could make it very challenging to identifying the modes only pertaining to the Artemis 1 integrated vehicle. While there is no current plan for modally decoupling the dynamics of the ML and Artemis 1 integrated test vehicle, several modal decoupling methods have been considered [10 - 28]. Hence a well correlated ML finite element model (FEM) is needed going into the IMT in order for not only an accurate modal pretest analysis, but to allow the model correlation focus to be on the Artemis 1 integrated vehicle. However, it should be kept in mind the ML FEM correlated to the ML Only modal test and subsequent ML on Transporter (CT)-2 rollout test data may have significant residual uncertainty because there is no launch vehicle mass loading of the ML Deck in either of these tests. Subsequent testing has been baselined by the programs (i.e., Space Launch System, Exploration Ground System, etc.) to gather ML Deck stiffness data that should help to reduce uncertainty in the correlated ML FEM.

As part of the risk reduction process, a building block approach [29] has been adopted and a key element is a modal test of the ML, referred to as the ML Only modal test. This modal test was performed in June 2019. This test will be vital in generating a well test correlated ML FEM. Immediately following the ML Only modal test, the ML on the CT-2 [30, 31] rolled out from the VAB to Launch Pad 39B and back, similar to a rollout performed in September 2018 and shown in Figure 5. The ML FEM will also be correlated to the June 2019 rollout test data using Operational Modal Analysis (OMA). CT-2 is a recently upgraded version of one of the original CT's used for the Apollo and Space Shuttle programs. The upgrades allow CT-2 to carry the heavier loading imposed by the integrated SLS launch vehicle and ML. It takes approximately eight hours for the CT-2 to transport SLS on the ML from the VAB to launch pad 39B. CT-2 is the size of a baseball infield, with a top speed of $1 \mathrm{mph}$ loaded and $2 \mathrm{mph}$ unloaded. CT-2 weights over 6 million pounds, height of between 20 feet and 26 feet depending on its jacking level, and is able to transport 18 million pounds. Figure 5 shows CT-2 rolling on the crawler way to Launch Pad 39B in March of 2016. A separate modal pretest analysis was performed for the ML on CT-2 rollout configuration [32] and will not be addressed in this paper. 
Because the ML structural dynamic properties are of paramount importance in accurately predicting rollout environment, separation dynamics during liftoff, and now serving as the dynamic modal test fixture for the IMT, the ML should be thought of as the "Oth stage" of the SLS launch vehicle and is a truly "complicated aerospace structure".

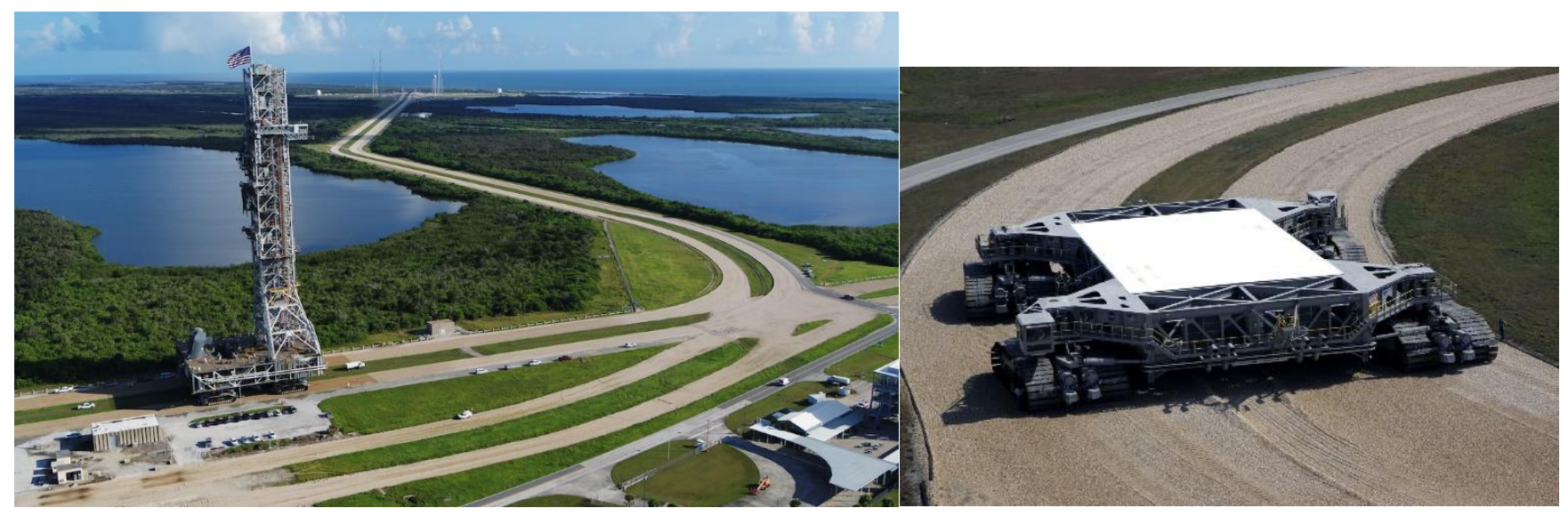

Figure 5. SLS Mobile Launcher Rolling Out to Launch Pad 39B, September 2018 (l) And CT-2 Rolling On Crawler Way (r).

This paper discusses the ML Only modal pretest analysis, which in addition to standard pretest analysis checks verifying adequacy of the exciter and accelerometer layout also included a force response analysis to generate "test like" acceleration time histories from which modal parameters were extracted. All the three test configurations were looked at: ML on the VAB Support Posts, ML on CT-2, and ML on the VAB Support Posts and CT-2. For the force response analysis to generate "test like" acceleration time histories, the planned instrumentation, shakers, excitation types, expected range of modal damping values, estimated ambient and sensor noise levels were used. These "test like" acceleration time histories were processed and had modes extracted using the same data processing software available to the test team. This "as-run end-toend" simulation aspect was done to ensure a high likelihood of successfully identifying the primary target modes.

\section{VERIFYING VALIDITY OF RESULTS}

A series of intermediate checks were built into the pretest analysis to support verifying the validity of the results. Model checks were performed on all FEM's, which included computing mass properties, free-free normal modes, fixed-base normal modes, $1 \mathrm{~g}$ static loading in three orthogonal directions, unit displacement in three orthogonal directions, and static loadings applied in all three orthogonal directions at all excitation locations. All intermediate checks were successfully completed.

Static loadings applied in all three orthogonal directions at all excitation locations were used to compute their local compliances. The shaker suitability study used force to acceleration (A/F) frequency response functions (FRF) synthesized from the FEM mode shapes and modal frequencies assuming 1\% modal damping. These drive point A/F FRF were double integrated in the frequency-domain to obtain the corresponding force to displacement (D/F) FRF, which were compared to the statically derived drive point compliances to verify their validity. Finally, the force response analysis generate both clean and noisy "test like" acceleration response time histories. These "test like" acceleration response time histories were then processed into A/F FRF and compared to those from the shaker suitability study to verify the validity of the force response analysis. Figure 6 shows this comparison for one of the shaker drive point locations. The sensor and ambient background noise effect the first three resonance speaks the most because the ML shaker force levels are lower in this frequency range due to their stroke limitations. 


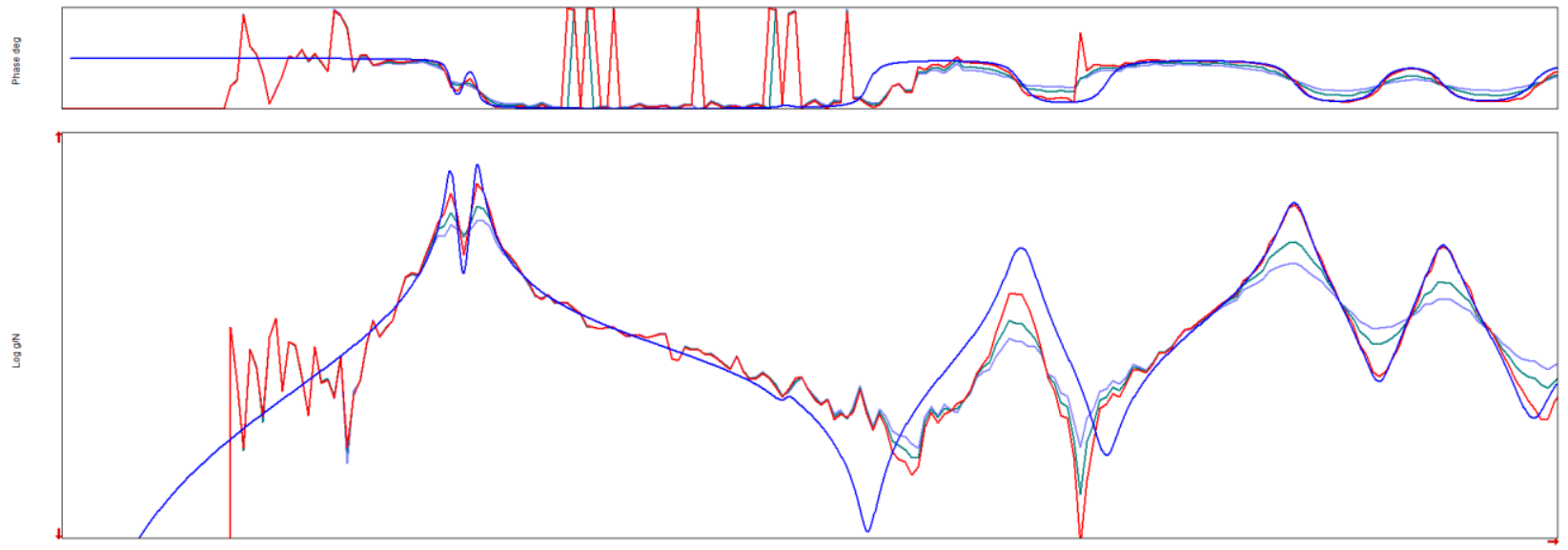

Figure 6. Drive Point A/F FRF Comparison: No Noise 1\% Damping (blue), Noisy 1\% Modal Damping (red), Noisy 2\% Modal Damping (green), Noisy $+3 \%$ Modal Damping (light blue).

\section{RESIDUAL VECTORS AND ACCOUNTING FOR COMPLIANCE CONTRIBUTION OF OUT-OF-BAND MODES}

Residual vectors are required to accurately account for the compliance contribution of the out-of-band modes in the pretest analysis. Including elastic body modes up to four times the frequency range of interest were retained, but without residual vectors, erroneously showed the shaker and drop hammer drive points were less compliant (i.e. stiffer) than what they actually are, which in turn lead to the erroneous conclusion they were able to excite more modes then they actually could. Figure 7 shows the effect of including residual vectors on the D/F drive point FRF at two locations, one that is very stiff and the other that has significant compliance. This shows that even if the frequency range of interest is quadrupled, but residual vectors are not included, the drive point compliance may have significant error (i.e. magnitude of the D/F FRF at $0 \mathrm{~Hz}$ does not match the statically derived drive point compliance). Modal damping values of $100 \%$ were used for all residual vectors in the force response analysis so that only their static elastic contribution and not their dynamic contribution was included.
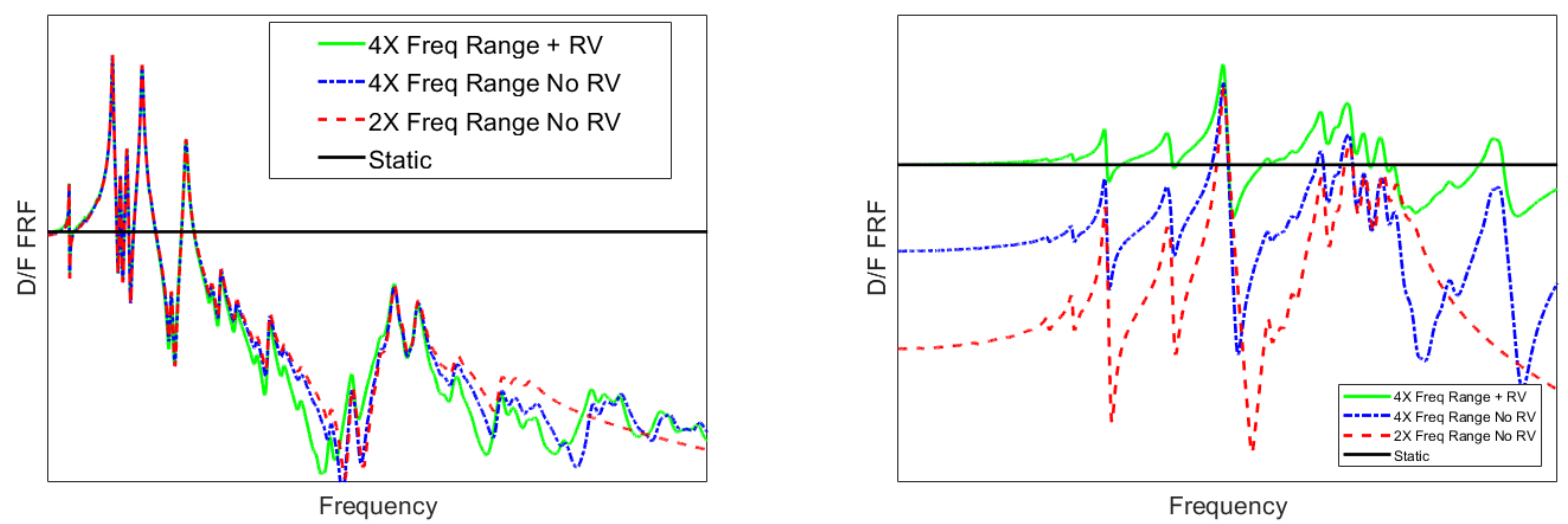

Figure 7. Residual Vectors Effect On Drive Point Compliance: Stiff Location (l) and Compliant Location (r). 


\section{TARGET MODE SELECTION}

Primary and secondary target mode selection plays a key role in a successful modal test, particularly when the testing schedule is tight. It can significantly help reduce the number of accelerometers and shakers and helps to focus the modal test team's mode extraction effort. Primary target modes are the critical "must have" modes needed to successfully correlate the FEM. Secondary target modes are modes of interest that provide useful information. The model correlation team should concur with the final target mode set and ideally be present during the modal test. Primary and secondary target modes selection uses modal effective mass and engineering judgement to capture fundamental characteristics of the ML and CT-2. For the ML on CT-2 and the ML on VAB Support Posts and CT-2 test configurations, the CT-2 has a significant amount of grounded mass due to the way its four trucks are modeled and constrained to ground. The pretest analyst should keep in mind how much of the total FEM mass is "locked" up in the boundary conditions and take this into account when determining a "small" modal effective mass fraction threshold. Figure 8 shows the cumulative modal effective mass for the ML on the VAB Support Posts, which has the typical characteristics of the majority of the modal effective mass being accounted for with the fundamental low frequency modes. As already mentioned, the ML Deck will not be mass loaded by a launch vehicle, which would have driven the ML Deck modes down in frequency. Because of this, modes of the ML Deck during this modal test, which exhibited significant vertical deflection, were retained as primary target modes even if their modal effective mass fraction was small or their modal frequency was slightly outside the original frequency range of interest. For all three test configurations, approximately two dozen total primary and secondary target modes were selected.
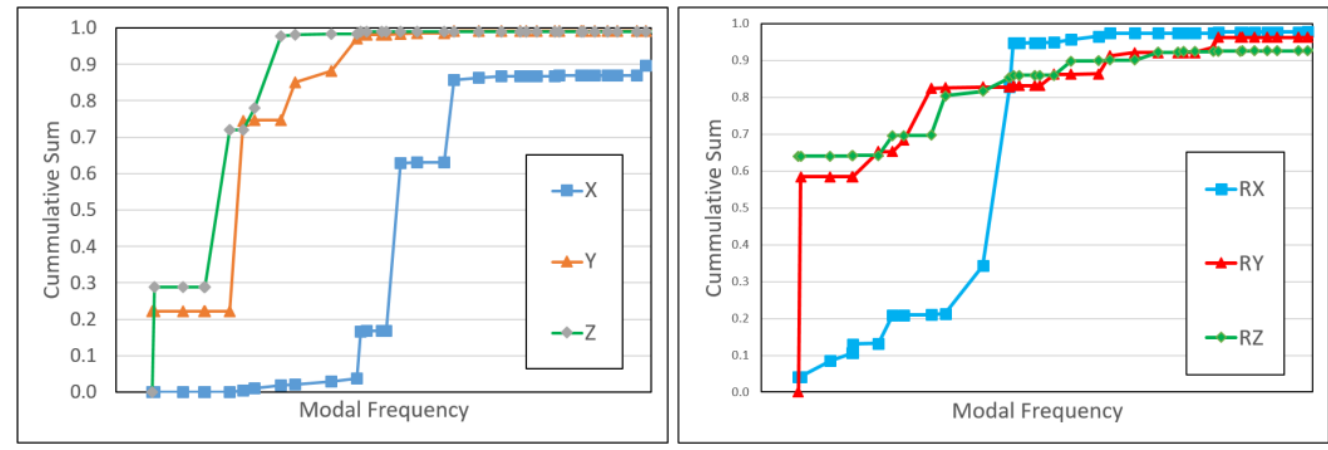

Figure 8. ML On VAB Support Posts Cumulative Modal Effective Mass: Translational (1) and Rotational (r).

\section{TEST DOF SELECTION}

The ML and CT-2 test DOF were determined during the course of several previous modal pretest analyses. Self and crossorthogonalities metrics were the criteria used to verify the ML and CT-2 test DOF were still adequate to identify all primary target modes. If the primary target modes self orthogonality off-diagonals magnitudes were $5 \%$ (10\% is a more common threshold) or less and the magnitudes of the off-diagonals of the cross orthogonality of the primary target modes and all FEM modes within the frequency range of interest were $5 \%$ (10\% is a more common threshold) or less, then the test DOF were judged to well (adequately) identify all primary target modes. However, the more rigorous criterion of 5\% was considered in order to obtain a "well correlated" ML FEM.

This pretest analysis showed the ML and CT-2 test DOF are well suited to identify all target modes in the frequency range of interest for all three test configurations. However, the ML test DOF by itself is not adequate to identify all target modes of the ML on CT-2 and ML on VAB Support Posts and CT-2 test configurations. Figure 9 shows the cross orthogonality of the primary and secondary target modes and all FEM modes in the frequency range of interest for the ML on CT-2 test configuration. This is not surprising since the CT-2 by itself has modes within the frequency range of interest, with its first mode being the vertical bounce mode of the CT-2 chassis. Hence, the CT-2 acts a large sprung mass attached to the underside of the ML. This pretest analysis did not show that shakers were required to drive directly on the CT-2 to adequately excite all primary target modes. Having additional shakers driving on the CT-2 would provide margin since both the ML and CT-2 FEM's are not test correlated, to mitigate issues due to the relative mass of the CT-2, and CT-2 modes coupling with those of the ML. If time had allowed, prioritizing the test DOF would have provided insight into how sensitive the modal test is to loss of test DOF and to identify key test DOF for which testing must be halted until repaired. 
While this pretest analysis showed that test DOF were not required at the boundary of the ML and VAB Support Posts, test DOF should always be located at the boundaries to verify boundary conditions even if not needed from a self/crossorthogonality perspective. Not having test DOF at the boundary can lead to erroneously changing the test article to make up for unexpected compliance/dynamics in the boundary condition.

The final checks of the adequacy of the selected test DOF is displaying them on the FEM and animating the target mode shapes with a Test Display Model (TDM), which has grid points only where test DOF are located. If the distribution of the test DOF on the FEM or the animations of the target mode using the TDM do not make sense, then a revision to the test DOF is required.

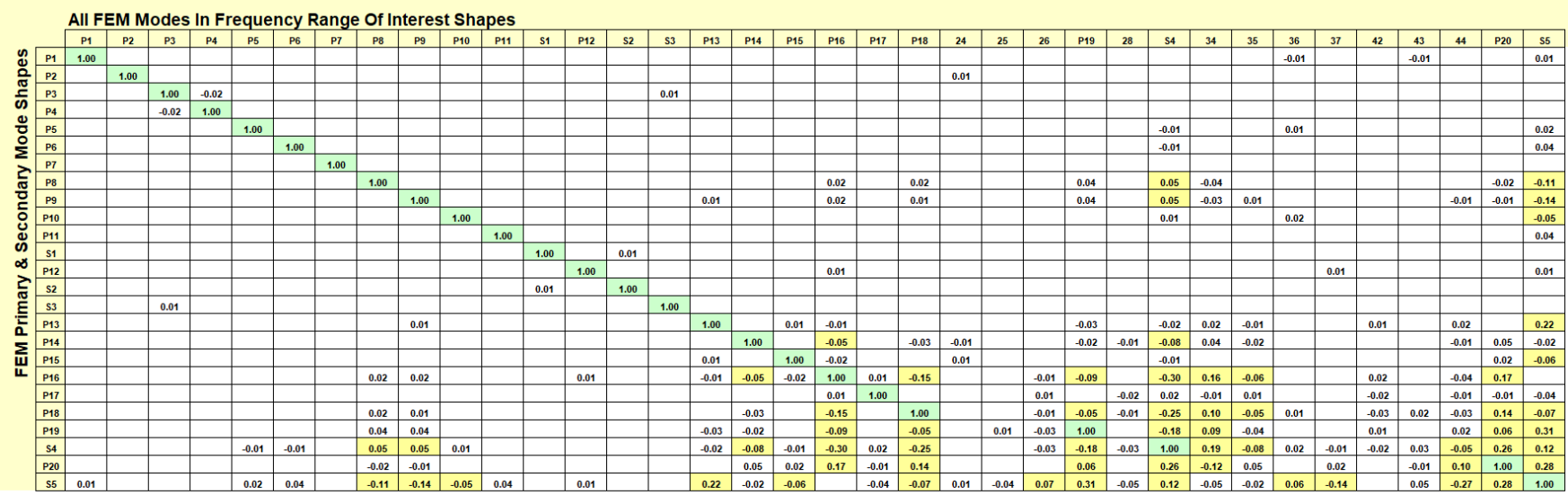

Off-diagonal entries having magnitudes less than 0.01 not shown.

Off-diagonal entries having magnitudes greater than or equal to 0.05 are highlighted in yellow.

Figure 9. ML on CT-2 Test Configuration Cross Orthogonality.

\section{SHAKERS}

The ML Only modal test shakers are inertial reaction mass shakers. There were three lateral (horizontal) ML shakers with their inertial reaction mass riding on bearing rails and two vertical ML shakers with their inertial reaction mass moving on vertical guide-posts. NASA/Marshall Space Flight Center (MSFC) test personnel designed and fabricated the ML shakers and incorporated the hydraulic actuators from their modal shakers used during the Ares I-X Flight Test Vehicle (FTV) modal test in 2009 [33 - 37]. Two lateral ML shakers were located on the ML Tower and two vertical and one lateral ML shaker were located on the ML Deck

Because the ML shakers are inertial reaction mass shakers, they have a corner frequency for which their peak force decreases for frequencies below this corner frequency and is proportional to frequency squared, due to shaker stroke length limits. Unfortunately, the ML shakers "knee" frequency was significantly above the lowest frequency primary target modes resulting in the ML shakers having limited force input into the lowest frequency primary target modes.

Figure 10 shows the normal probability plot of the fourth ML shaker force time history MSFC engineers provided. Even though the shaker drive signal was a bandwidth limited Gaussian signal, the ML shaker force exhibits significant nonGaussian behavior, which is due to the nonlinearities in the hydraulic actuators. 


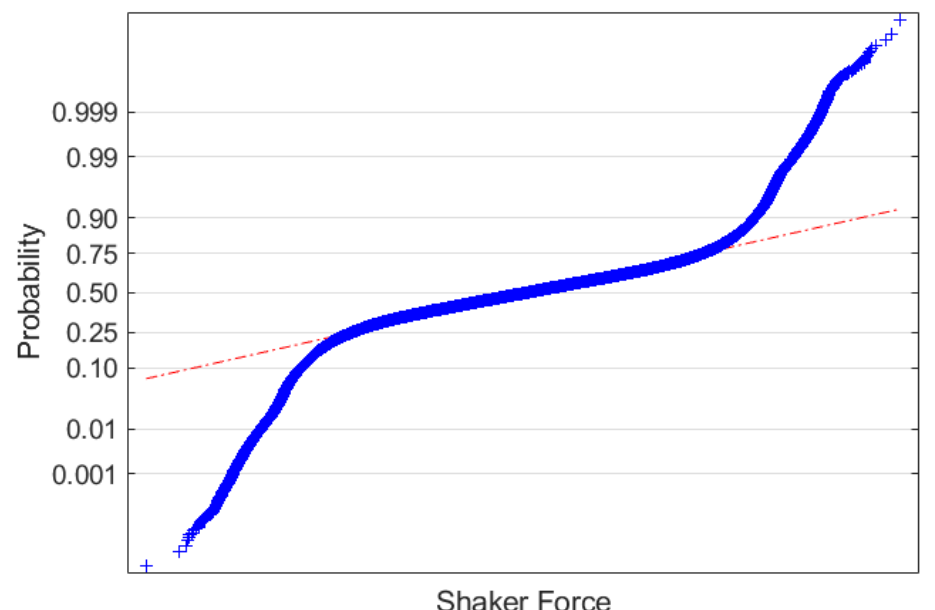

Figure 10. ML Shaker Force \#4 Time History Normal Probability Plot.

These ML shaker forces were at a higher sampling frequency than used in the modal pretest force response analysis. They were appropriately lowpass filtered and down sampled to the modal pretest force response analysis sampling frequency and verified by comparing power spectral density (PSD). Figure 11 shows the Autocorrelation functions computed on all four MSFC provided ML shaker force time histories, which shows that correlation essentially ceases to exist for time lags greater than or equal to five seconds.

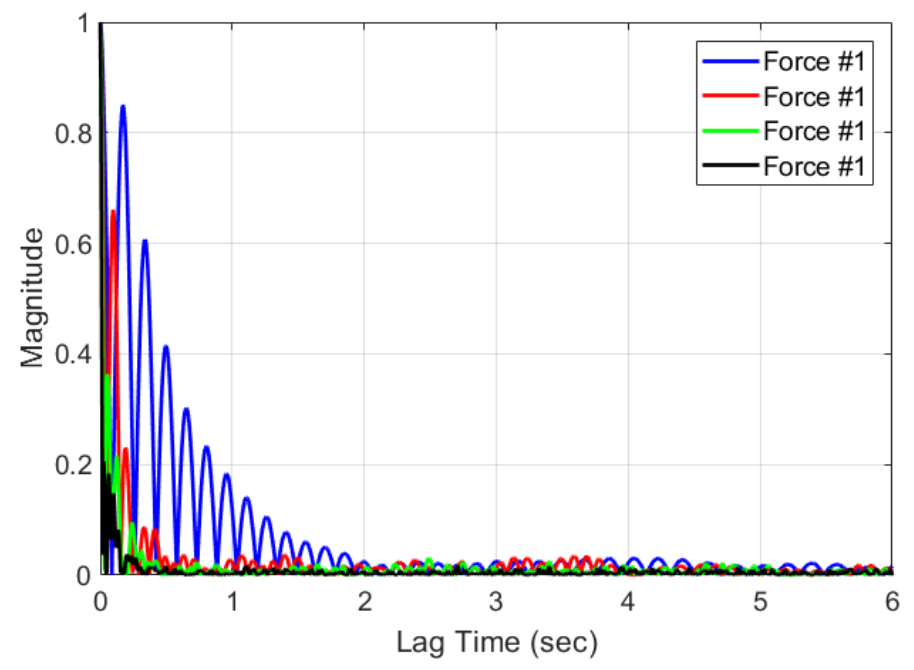

Figure 11. Autocorrelation Function Of ML Shaker Forces.

From each MSFC provided ML shaker force time history, five uncorrelated ML shaker force time histories were generated for use in the modal pretest Multi-Input Multi-Output (MIMO) force response analysis. Since the data processing frame length used to process the force response analysis time histories into PSD, FRF, and coherence significantly exceeded five seconds, a "Slinky Approach" was used to generate five uncorrelated shaker force time histories. ML shaker \#1 force time history was created by taking the original recorded lateral shaker force time history and appending it to itself as many times as necessary until the assembled time duration equaled or exceeded the desired time duration. ML shaker \#2 force time history was generated by lopping off the first data processing frame length of ML Shaker \#1 time history and appending it to its end. This process repeated for the remaining ML shaker force time histories. Because the data processing frame length was significantly longer than five seconds, lopping off only first five seconds would have end up with the five ML random 
shaker force time histories being correlated and having very high coherences. This is due to each ML shaker force time history essentially being time delayed versions of themselves [38].

\section{DROP HAMMER FORCES}

The MSFC test group designed and fabricated a Drop Hammer to supplement the ML shakers excitation of the ML Deck to help identify higher frequency ML Deck primary target modes. The Drop Hammer can shape its impulsive force, both in terms of peak force and frequency bandwidth, by adjusting its drop height and shock absorber. MSFC test personnel recorded an impulse train from four drops of the Drop Hammer in their laboratory. These four impulse Drop Hammer force time history, after removing transients due to resetting of the Drop Hammer between drops, were converted into a 16 impulse Drop Hammer force time history having the same sampling frequency as the modal pretest analysis force response analysis. Validity of the 16 impulse Drop hammer force time histories was verified by comparison of its PSD's to that of the MSFC supplied four impulse Drop Hammer force time history.

\section{SHAKER AND DROP HAMMER SUITABILITY STUDY}

This study was performed to determine if the locations and orientations of the ML shakers and Drop Hammer were adequate to excite all target modes. FRF were synthesized from the FEM modes assuming $1 \%$ modal damping, since this was believed to be the lowest modal damping level present during the ML Only modal tests. A spectral resolution equal to 1/10th the half power bandwidth of the lowest frequency target mode was selected to ensure all resonance peaks in the FRF were well captured. Normal Mode Indicator Functions (NMIF) and Multivariate Mode Indicator Functions (MMIF) were computed on these synthesized FRF to determine if the ML shakers and Drop Hammer were adequate to excite all of the target modes. NMIF and MMIF values below 0.25 indicated potentially well excited target modes, and between 0.25 and 0.4 indicated potentially adequate excitation. This study did not look at ML shaker and Drop Hammer excitation levels. This study showed that the ML shakers and Drop Hammer locations and orientations are adequate to excite all target modes.

\section{ACCELEROMETER SENSOR NOISE}

Accelerometer sensor noise models were based upon the accelerometer manufacture's specification data sheets. The ML Only modal test used several different accelerometer models. For accelerometers having sensor noise specified as "broadband resolution" as a PSD in units of grms $2 / \mathrm{Hz}$, their sensor noise time histories was modeled as uncorrelated zero mean Gaussian random time histories having the correct spectral content using a Fast Fourier Transform (FFT) approach. For accelerometers having sensor noise specified as "resolution" in terms g's, their sensor noise time histories were modeled as uncorrelated random time histories uniformly distributed on the interval of $+/-\alpha \mathrm{g}$, where $\alpha \mathrm{g}$ was the specified resolution. For the accelerometers having sensor noise specified as "broadband resolution" only in terms of overall grms level, their sensor noise time histories were modeled as uncorrelated zero mean Gaussian random variables with a standard deviation equal to the "broadband resolution".

\section{AMBIENT BACKGROUND NOISE (NOT INCLUDING ACCELEROMETER AND DATA ACQUISITION SENSOR/SIGNAL NOISE)}

Ambient background noise is comprised of ambient vibrations and electromagnetic interference effects (e.g., $60-\mathrm{Hz}$ harmonics due to lighting, transformers, etc.). Ambient background vibration levels of the ML inside the VAB were not available for this pretest analysis. Instead, ambient dated collected during the Ares I-X Flight Test Vehicle (FTV) modal test, with the VAB doors closed, was used to estimate the ML ambient background noise levels [33 - 37]. Figure 12shows the Ares I-X FTV inside the VAB. 


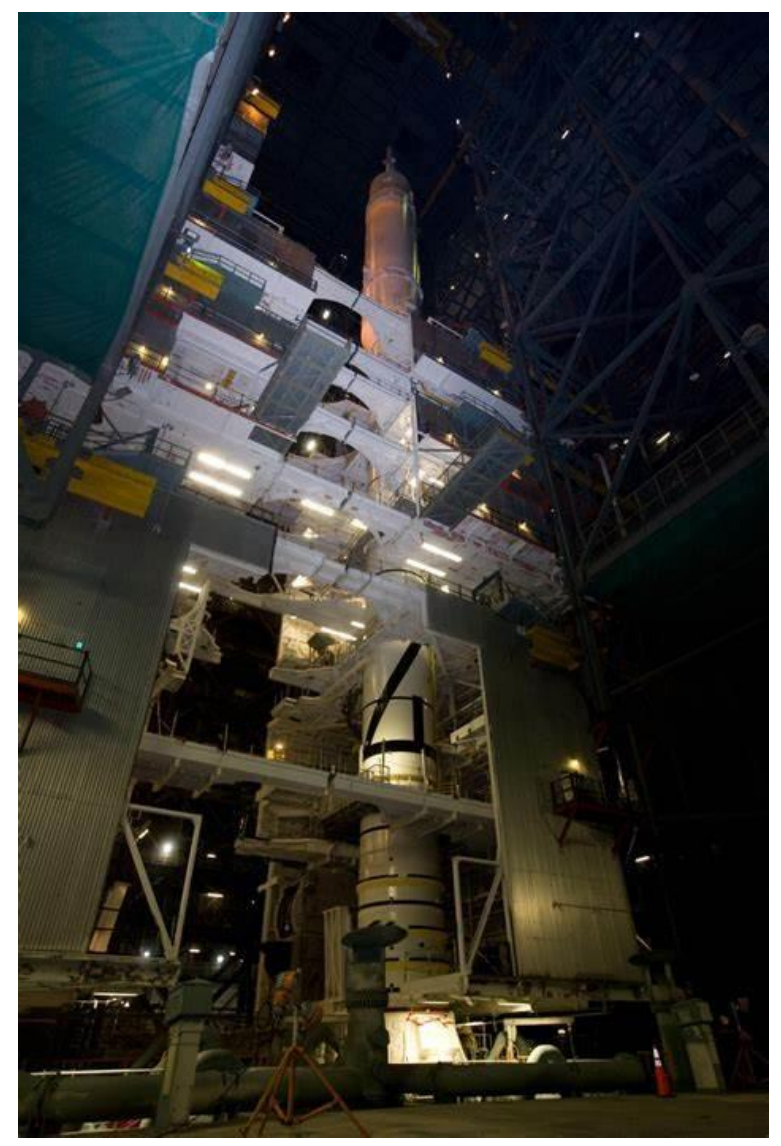

Figure 12. Ares I-X FTV Inside VAB.

These ambient acceleration time histories were highest near the top of the Ares I-X FTV and smallest on the Mobile Launch Platform (MLP) to which the Ares I-X FTV was mounted to. PSD computed on these ambient acceleration time histories clearly showed resonance peaks corresponding to modes of Ares I-X FTV and the MLP (i.e. system modes). The overall maximum, overall average, and overall minimum PSD were compared to the manufacturer's sensor noise spectrum for the accelerometers used during this modal test. The overall maximum PSD and the manufacturer's sensor noise spectrum showed good agreement, with the exception the overall maximum PSD having exceedances due to the Ares I-X FTV system modes. The ML ambient background noise was chosen to have a constant magnitude PSD that envelopes the accelerometer sensor noise spectrum and the overall average PSD magnitude of the Ares I-X ambient acceleration time histories, except for a few small exceedances of the resonance peaks due to the Ares I-X FTV system modes. The ML ambient background noise time histories were modeled as uncorrelated zero mean Gaussian random time histories. As forward work, it will be important to compare this assumed ambient background noise level to the actual ambient background noise levels to increase the accuracy of the subsequent IMT pretest analysis.

\section{FORCE RESPONSE ANALYSIS THEORY AND IMPLEMENTATION}

The modal pretest force response analysis utilized an open-loop MIMO modal state-space approach when using the five uncorrelated random ML shaker force time histories and a Single-Input Multiple-Output (SIMO) modal state-space approach when using the drop hammer force time histories. Equation 1 shows the modal state-space model used to generate acceleration response time histories. 


$$
\begin{aligned}
& M \ddot{x}(t)+C \dot{x}(t)+K x(t)=F(t), \Phi^{T} M \Phi \ddot{q}(t)+\Phi^{T} C \Phi \dot{q}(t)+\Phi^{T} K \Phi q(t)=\Phi^{T} F(t) \\
& \ddot{q}(t)+C C \dot{q}(t)+K K q(t)=\Phi^{T} F(t),\left[\begin{array}{l}
\dot{q}(t) \\
\ddot{q}(t)
\end{array}\right]=\left[\begin{array}{cc}
0_{n} & I_{n} \\
-K K & -C C
\end{array}\right]\left[\begin{array}{c}
q(t) \\
\dot{q}(t)
\end{array}\right]+\left[\begin{array}{l}
0_{n} \\
\Phi^{T}
\end{array}\right] F(t) \\
& {[\ddot{q}(t)]=\left[\begin{array}{ll}
-K K & -C C
\end{array}\right]\left[\begin{array}{l}
q(t) \\
\dot{q}(t)
\end{array}\right]+\Phi^{T} F(t), \quad[\ddot{x}(t)]=\Phi[\ddot{q}(t)]=\Phi[-K K \quad-C C]\left[\begin{array}{l}
q(t) \\
\dot{q}(t)
\end{array}\right]+\Phi^{T} F(t)}
\end{aligned}
$$

Equation 1. Force To Acceleration Modal State-Space Model.

Three modal damping levels of $1 \%, 2 \%$, and 3\%, were investigated for all three test configurations as it was believed they covered the range of modal damping that could be present during testing. The modal state-space model generated "clean" acceleration response time histories to which the ambient background noise and sensor noise acceleration time histories were then added in order to obtain the "test like" acceleration response time histories. The validity of the "clean" and "test like" acceleration response time histories was verified by comparing their drive point force to acceleration (A/F) FRF to the A/F FRF computed from the ML shaker and Drop Hammer suitability study. The ML shaker drive point displacements and velocities showed that neither the shaker displacement nor velocity limits were exceeded and thus verified this open-loop approach was valid and that a close-loop simulation involving detailed modeling of ML shakers was not needed.

These "test like" acceleration response time histories were processed into PSD, FRF, and coherence using the same analysis tools available to the test team. For the random shaker test runs, the time histories were block processed with a Hanning window, 90\% overlap, and a rather large frame length (i.e. block size), which was necessitated by a very fine spectral resolution needed to identify very closely spaced modes. For the drop hammer test runs, the time histories were similarly block processed, except no window or overlapping were applied.

These "test like" PSD, FRF, and coherence were then treated just like actual test data would be. Standard frequency-domain data quality checks were performed, which included overlaying the shaker force PSD, drive point coherence, and drive point A/F FRF; and computing coherences between the ML shaker force time histories to verify they were uncorrelated. Force response analysis with the ML shaker random forces and 1\% modal damping, as expected, clearly showed the effect of the Hanning window on the distortion of the low frequency resonance peaks in the FRF. Also as expected, significant dropouts in the coherence at frequencies close to the A/F FRF resonance peak frequencies were clearly evident.

The next step computed Multivariate Mode Indicator Function (MMIF) and or Complex Mode Indicator Function (CMIF) on selected FRF to obtain an estimate of the number of modes within the frequency range of interest. Then time-domain polyreference techniques extracted the majority of the test modes, with a Single Degree Of Freedom (SDOF) polynomial curve fitting tool used a few times. Advanced modal extraction techniques, such as mode enhancement and spatial filtering, were not used. Figure 13 shows a CMIF overlaid with pole estimates forming well behaved race track patterns indicating the test modes are straight forward to extract.

Self and cross-orthogonality criteria were used to determine how well the "test" primary target modes could be identified. If the magnitudes of the off-diagonal of the self orthogonality of the "test" primary target modes and the cross orthogonality of the "test" and FEM primary target modes being 5\% (10\%) or less, and the magnitudes of the diagonals of the cross orthogonality of the "test" and FEM primary target modes being $95 \%(90 \%)$ or greater, then the "test" primary target modes were well (adequately) identified. Figure 14 shows the cross orthogonality between all identified "test" modes and FEM modes in the frequency range of interest with the ML having $1 \%$ modal damping and sensor and ambient background noise included. Figure 15 shows the corresponding cross orthogonality between just the identified "test" primary target modes and all FEM modes in the frequency range of interest. This was looking at a subset of the accelerometers showing they will adequately identify the first 11 primary target modes. 


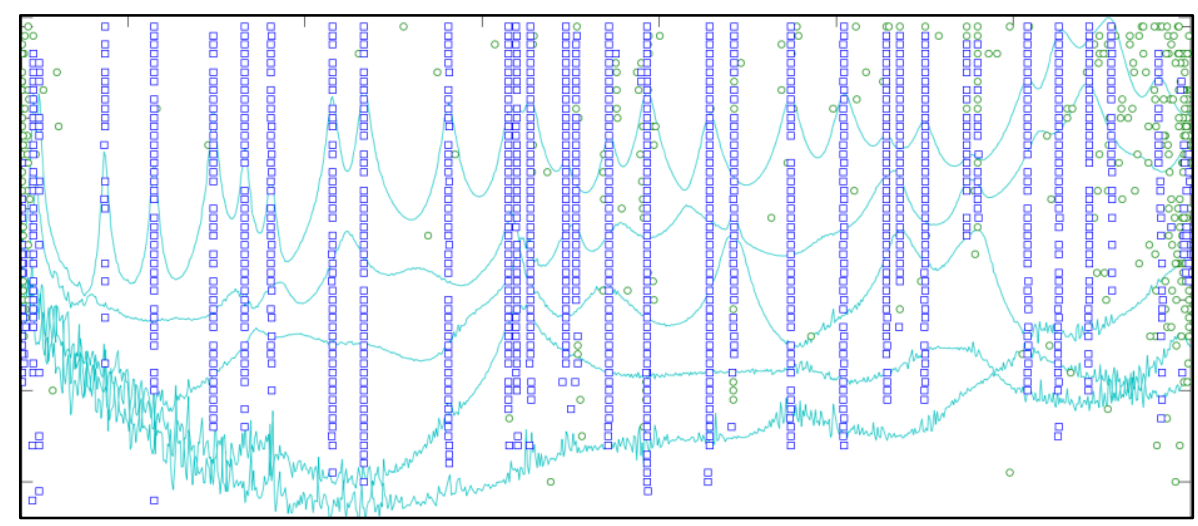

Figure 13. CMIF Overlaid With Pole Estimates.

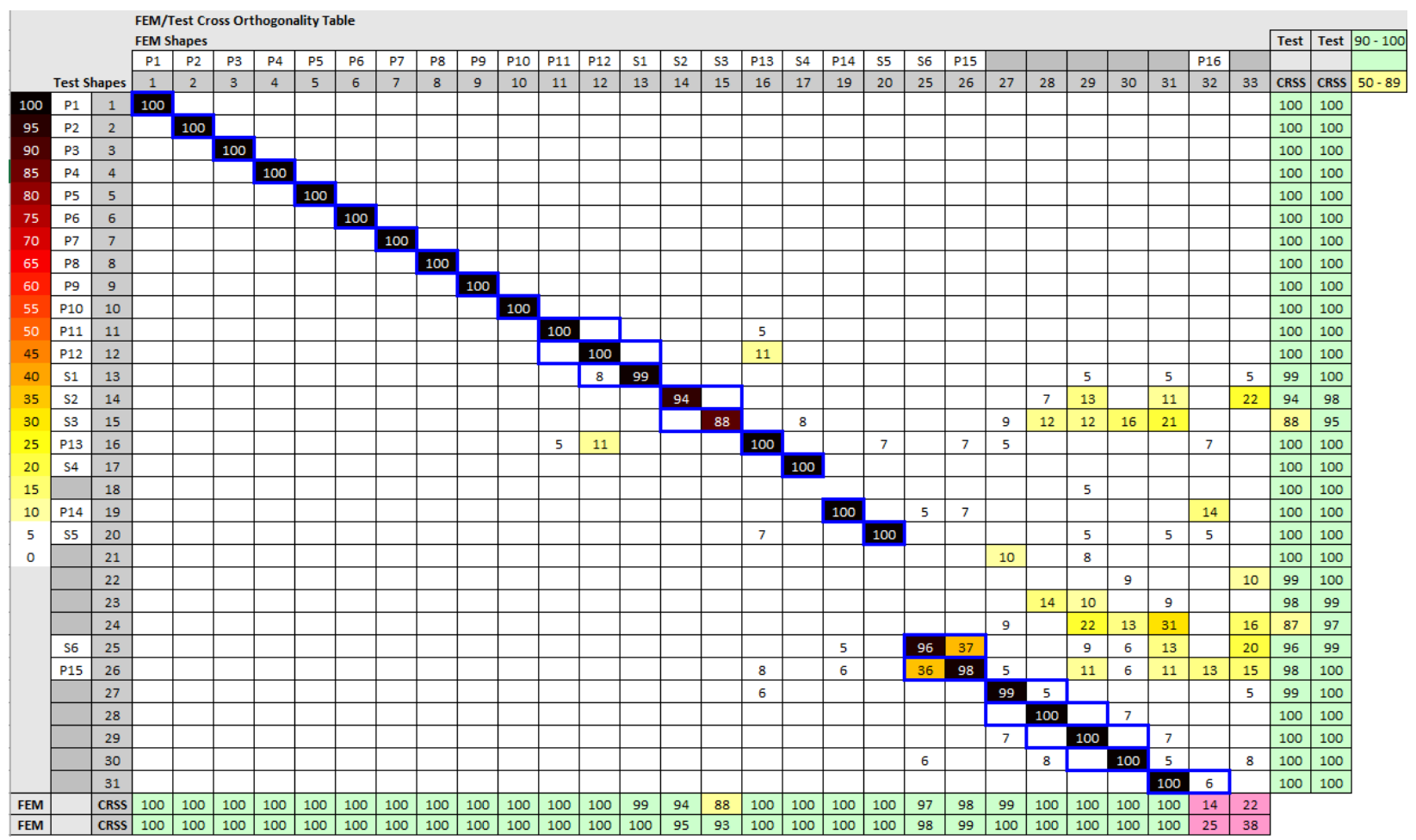

Figure 14. Test vs FEM Cross Orthogonality.

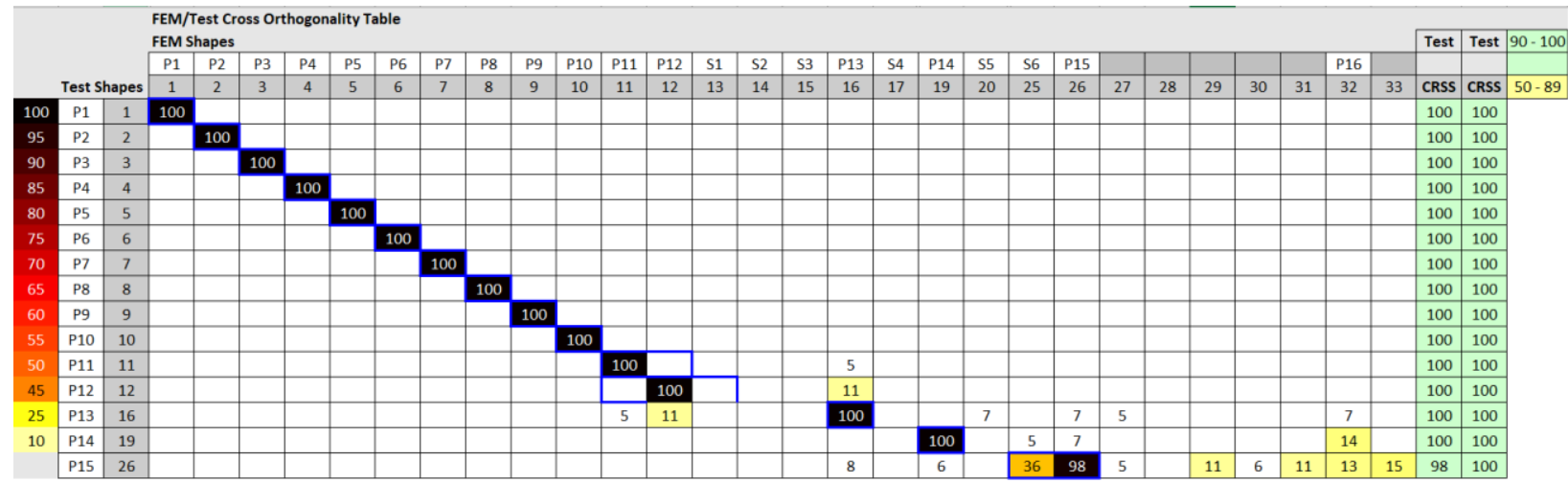

Figure 15. Test Primary Target Modes vs FEM Cross Orthogonality. 


\section{CONCLUSIONS}

This pretest analysis showed the primary target modes for all three test configurations can be adequately identified, and many times well identified for $1 \%-2 \%$ modal damping values. However, this requires running the ML shakers at their full force levels. Running the ML shakers at $50 \%$ and $20 \%$ of their full levels was investigated to determine if multi-force linearity studies (i.e. shakers run at 20\%,50\%, 100\% levels) were feasible. This type of linearity study is especially important to conduct during ML Only modal test since the ML Deck is not being mass loaded as it will be during the IMT. This pretest analysis predicts that running the ML shakers at $20 \%$ of their full level is insufficient if the modal damping is $3 \%$ or greater. This is due to ML shakers operating as inertial reaction mass shakers, the lowest frequency primary target modes being significantly below the corner frequency, and therefore the shaker force levels at the lowest frequency primary target modes being too low to generate acceleration responses levels sufficiently above the ambient and sensor noise levels. Hence multiforce level linearity studies may require multi-point sine sweeps $[39,40]$ or normal mode tuning [41] techniques.

The recognized challenge with modal pretest analysis is it utilizes uncorrelated FEM's, includes assumptions about the ambient and accelerometer sensor noise levels, and most importantly assumed the ML and CT-2 behave linearly. Therefore, margin needs to be included in the test planning and preparation, and most importantly during the ML Only modal test there should be technical flexibility and capability to make the necessary adjustments to shaker, hammer, and instrumentation locations/orientations to ensure identifying all primary target modes.

\section{REFERENCES}

[1] "SLS Fact Sheet.", https://www.nasa.gov/exploration/systems/sls/factsheets.html.

[2] "NASA Facts: Space Launch System.”, https://www.nasa.gov/sites/default/ files/files/SLS-Fact-Sheet_aug2014finalv3.pdf.

[3] "Environmental Assessment: Modification and Operation of Test Stand 4550 in Support of Integrated Vehicle Ground Vibration Testing for the Constellation Program Marshall Space Flight Center, Contract No. NNM05AB44C, Task Order No. CH338.”, https://www.nasa.gov/pdf/247125main_MSFC_TS4550_ EA Final.pdf.

[4] “Saturn V Dynamic Test Vehicle.”, https://en.wikipedia.org/wiki/Saturn_V_Dynamic_Test_Vehicle.

[5] Lemke, P., Tuma M., and Askins B., "Integrated Vehicle Ground Vibration Testing of Manned Spacecraft: Historical Precedent.”, https://ntrs.nasa.gov/ archive/nasa/casi.ntrs.nasa.gov/20080031432.pdf

[6] "NASA History, This Week in NASA History: Space Shuttle Program's first Mated Vertical Ground Vibration Test Performed at Marshall - Oct 4, 1978.”, https://www.nasa.gov/centers/marshall/history/this-week-in-nasahistory-space-shuttle-program-s-first-mated-vertical-ground.html.

[7] Henry, K., "Getting to Know you, Rocket Edition: Interim Cryogenic Propulsion Stage.", https://www.nasa.gov/sls/interim_cryogenic_propulsion_stage 141030.html.

[8] "Mobile Launcher, NASA Fact Sheet." https://www.nasa.gov/sites/default/files/ atoms/files/mobilelauncher_ factsheet_v2.pdf.

[9] "Mobile Launcher Umbilicals and Support, NASA Facts." https://www.nasa.gov/ sites/default/files/atoms/files/ml_umbilicals20160523.pdf.

[10] Kerrian, P., Napolitano, K., "Pretest Analysis for Modal Survey Tests Using Fixed Base Correction Method." Proceedings of the $37^{\text {th }}$ International Modal analysis Conference, 2019.

[11] Napolitano, K., "Maximizing the Quality of Shape Extractions from Base Shake Modal Tests." Proceedings of the $37^{\text {th }}$ International Modal analysis Conference, 2019.

[12] Napolitano, K., "Fixing Degrees of Freedom of an Aluminum Beam by Using Accelerometers as References." Proceedings of the $37^{\text {th }}$ International Modal analysis Conference, 2019.

[13] Napolitano, K., N. Yoder, Fladung, W. "Extraction of Fixed-Base Modes of a Structure Mounted on a Shake Table." Proceedings of the 31 International Modal Analysis Conference, 2013.

[14] Napolitano, K., and N. Yoder. "Fixed Base FRF Using Boundary Measurements as References - Analytical Derivation." Proceedings of the 30 ${ }^{\text {th }}$ International Modal Analysis Conference, 2012. 
[15] Allen, M., "Recent Advances to Estimation of Fixed-Interface Modal Models using Dynamic Substructuring," Proceedings of the $36^{\text {th }}$ International Modal Analysis Conference, 2018.

[16] Allen, M., Gindlin, H., and Mayes, R., "Experimental Modal Substructuring to Estimate Fixed-Base Modes from Tests on a Flexible Fixture.” Journal of Sound and Vibration 330 (2011), 4413-4428.

[17] Kaufmann, D., and Majed, A., “Accelerance Decoupling (AD) Method,” NESC-RP-17-01207.

[18] Crowley, J., Klosterman, A., Rocklin, G., and Vold, H., "Direct Structural Modification Using Frequency Response Functions", Proceedings of the $2^{\text {nd }}$ International Modal Analysis Conference, 1984, 58-65.

[19] Beliveau, J.G., F.R. Vigneron, Y. Soucy, and S. Draisey. "Modal Parameter Estimation from Base Excitation." Journal of Sound and Vibration 107. 1986: 435-49.

[20] M. Imregun, D. A. Robb, and D. J. Ewins, "Structural Modification and Coupling Dynamic Analysis Using Measured FRF Data", $5^{\text {th }}$ International Modal Analysis Conference (IMAC V), London, England, 1987.

[21] Napolitano, K., Winkel, J., Akers, J., Suarez, V., and Staab, L. "Modal Survey of the MPCV Orion European Service Module Structural Test Article Using a Multi-Axis Shake Table." Proceedings of the $36^{\text {th }}$ International Modal Analysis Conference, 2018.

[22] Staab, L., J. Winkel, V. Suárez, T. Jones, and K. Napolitano. "Fixed Base Modal Testing Using the Mechanical Vibration Facility 3-Axis Base Shake System." Proceedings of the $34^{\text {th }}$ International Modal Analysis Conference, 2016.

[23] Carne, T.G., D.R. Martinez, and A.R. Nord. "A Comparison of Fixed-Base and Driven Base Modal Testing of an Electronics Package." Proceedings of the $7^{\text {th }}$ International Modal Analysis Conference, 1989: 672-9.

[24] Beliveau, J.G., F.R. Vigneron, Y. Soucy, and S. Draisey. "Modal Parameter Estimation from Base Excitation." Journal of Sound and Vibration 107. 1986: 435-49.

[25] Fullekrug, U. "Determination of Effective Masses and Modal Masses from Base-Driven Tests." Proceedings of the $14^{\text {th }}$ International Modal Analysis Conference, 1996: 671-81.

[26] Sinapius, J.M. "Identification of Fixed and Free Interface Normal Modes by Base Excitation." Proceedings of the $14^{\text {th }}$ International Modal Analysis Conference, 1996: 23-31.

[27] Mayes, R.L., and L.D. Bridgers. "Extracting Fixed Base Modal Models from Vibration Tests on Flexible Tables." Proceedings of the $27^{\text {th }}$ International Modal Analysis Conference, 2009.

[28] Mayes, R., D. Rohe, and J. Blecke. "Extending the Frequency Band for Fixed Base Modal Analysis on a Vibration SlipTable." Proceedings of the 31 $1^{\text {st }}$ International Modal Analysis Conference, 2013.

[29] Sills, J. and Allen, M., "Historical Review of 'Building Block Approach' in Validation for Human Space Flight." Proceedings of the $37^{\text {th }}$ International Modal Analysis Conference, 2019.

[30] "Ground Systems Development \& Operations Highlights, March 2017." https://www.nasa.gov/sites/default/files/atoms/files/gsdohighlights march2017.pdf.

[31] “Crawler-Transporter 2, NASA Fact Sheet." https://www.nasa.gov/sites/default/ files/atoms/files/crawlertransporters-2-oct2016.pdf.

[32] Winkel J., Akers J., and Bruno E., "Feasibility Study of SDAS Instrumentation's Ability to Capture Mobile Launcher/Crawler-Transporter Target Modes During Rollout Operations.", Proceedings of the $38^{\text {th }}$ International Modal Analysis Conference, 2020.

[33] Buehrle, R., Templeton, J., Reaves, M., Horta, L., Gaspar, J., Barolotta, P., Parks, R., and Lazor, D., “Ares I-X Flight Test Vehicle Modal Test." NASA/TM-2010-216182.

[34] Buehrle, R., Templeton, J., Reaves, M., Horta, L., Gaspar, J., Barolotta, P., Parks, R., and Lazor, D., “Ares I-X Flight Test Vehicle: Stack 1 Modal Test.” NASA/TM-2010-216210.

[35] Buehrle, R., Templeton, J., Reaves, M., Horta, L., Gaspar, J., Barolotta, P., Parks, R., and Lazor, D., “Ares I-X Flight Test Vehicle: Stack 5 Modal Test.” NASA/TM-2010-216183.

[36] Buehrle, R., Templeton, J., Reaves, M., Horta, L., Gaspar, J., Barolotta, P., Parks, R., and Lazor, D., “Ares I-X Launch Vehicle Modal Test Overview." Proceedings of the 27th International Modal Analysis Conference, 2010.

[37] Buehrle, R., Templeton, J., Reaves, M., Horta, L., Gaspar, J., Barolotta, P., Parks, R., and Lazor, D., “Ares I-X Launch Vehicle Modal Test Measurements and Data Quality Assessments." Proceedings of the $27^{\text {th }}$ International Modal Analysis Conference, 2010. 
[38] Paez Wirching, P., Paez, T., Ortiz, K., Random Vibrations: Theory and Practice, Wiley, New York, Section 5.2.2, 141.

[39] Napolitano, K., Yoder, N., "Optimal Phasing Combination for Multiple Source Excitation.” Proceedings of the $31^{\text {st }}$ International Modal Analysis Conference, 2014.

[40] Napolitano, K., Linehan, D., "Multiple Sine Sweep Excitation for Ground Vibration Tests." Proceedings of the $27^{\text {th }}$ International Modal Analysis Conference, 2009.

[41] Hunt, D.L., J. Matthews, and R. Williams, "An Automated Tuning and Data Collection System for Sine Dwell Modal Testing," $25^{\text {th }}$ Structures, Structural Dynamics and Materials Conference and AIAA Dynamics Specialists Conference, Palm Springs, CA, May, 1984. 